\title{
High energy particles accelerated during the large solar flare of 1990 May 24: X/ $/ \gamma$-ray observations
}

\author{
N. Vilmer ${ }^{1}$, A. L. MacKinnon ${ }^{2}$, G. Trottet ${ }^{1}$, and C. Barat ${ }^{3}$ \\ 1 LESIA, UMR CNRS 8109, Observatoire de Paris, 5 place J. Janssen, 92195 Meudon, France \\ 2 Dept of Adult and Continuing Education, University of Glasgow, Glasgow G12 8QQ, UK \\ ${ }^{3}$ Centre d'Étude Spatiale des Rayonnements, CNRS/UPS, BP 4346, 31029 Toulouse, France
}

Received 4 April 2003 / Accepted 16 September 2003

\begin{abstract}
The PHEBUS experiment aboard GRANAT observed $\gamma$-ray line emission and $\gamma$-ray continuum above $10 \mathrm{MeV}$ from the 24 May, 1990 solar flare. Observations and interpretation of the high-energy continuum have been discussed previously. Here we re-examine these, combining the PHEBUS observations above $10 \mathrm{MeV}$ with calculations of the pion decay continuum to quantitatively constrain the accelerated ion energy distribution at energies above $300 \mathrm{MeV}$. The uncertainty in the determination of the level of the primary electron bremsstrahlung as well as the lack of measurements on the $\gamma$-ray emission above $\simeq 100 \mathrm{MeV}$ combine to allow rather a wide range of energy distribution parameters (in terms of the number of protons above $30 \mathrm{MeV}$, the spectral index of the proton distribution and the high energy cut-off of the energetic protons). Nevertheless we are able to rule out some combinations of these parameters. Using the additional information provided by the $\gamma$-ray line observations we discuss whether it is possible to construct a consistent picture of the ions which are accelerated in a wide energy range during this flare. Our findings are discussed with respect to previous works on the spectrum of energetic protons in the $10 \mathrm{MeV}$ to $\mathrm{GeV}$ energy range.
\end{abstract}

Key words. Sun: activity - Sun: flares - Sun: X-rays, gamma rays

\section{Introduction}

Ground-based cosmic ray studies told us, fifty years ago, that solar flares are capable of accelerating protons to $\mathrm{GeV}$ energies. Solar $\gamma$-ray astronomy, however, opened up a new window for the study of these extremes of solar particle acceleration, particularly through the detection of secondary energetic neutrons, and of pion decay $\gamma$-ray continuum. Resulting from reactions involving primary flare particles with energies of $>50 \mathrm{MeV} /$ nucleon and $>300 \mathrm{MeV} /$ nucleon, respectively, these two radiations combine with de-excitation gamma-ray lines to yield, in principle, a fairly complete picture of the accelerated ion distribution above a few MeV/nucleon (e.g. Chupp 1984; Chupp 1996; Ramaty 1986; Trottet \& Vilmer 1997; Vilmer \& MacKinnon 2003 for reviews). Detailed study of high-energy ions at the flare site starts with SMM (Solar Maximum Mission) (Murphy et al. 1987), goes on with events observed with GAMMA1 (Akimov et al. 1992), CGRO (Compton Gamma Ray Observatoty (e.g. Mandzhavidze \& Ramaty 1992; Kanbach et al. 1993; Dunphy et al. 1999) and the GRANAT spacecraft (Debrunner et al. 1992, 1993, 1997). Observations of flares with detectable emission at these high energies are still rare, however, to the extent that each one deserves detailed scrutiny.

Pion decay radiation, together with observations of neutrons, provides specific information on the highest ion

Send offprint requests to: N. Vilmer,

e-mail: nicole.vilmer@obspm.fr energies produced during flares. Charged pions decay to yield electrons and positrons, which in turn produce $\gamma$-ray photons by bremsstrahlung. Positrons also contribute to the continuum by annihilating in flight. Superimposed on this resulting hard continuum, is a very flat, broad, "bump" feature which has a maximum at $67 \mathrm{MeV}$ and results from the decay to two photons of neutral pions. Synchrotron losses of the charged secondaries (electrons and positrons) may be important for typical solar atmosphere parameters. These shorten the radiatively effective lifetime of the electrons and positrons, depressing their continuum radiation relative to the $\pi^{0}$ feature (Murphy et al. 1987). We might hope to diagnose from spectrally-resolved observations the average field strength in the source region in this way, from the height of the $\pi^{0}$ feature above the continuum.

Flares can also accelerate primary electrons to energies $>10 \mathrm{MeV}$. These produce their own bremsstrahlung continuum at comparable energies, potentially masking pion decay radiation. Electron bremsstrahlung, however, must decline monotonically. Thus the spectrum of $\pi^{0}$ decay, with a spectral maximum at $67 \mathrm{MeV}$, cannot be mimicked by primary electron bremsstrahlung. Observations of the appropriate spectral hardening around this photon energy thus constitute an unambiguous diagnostic for high-energy proton acceleration. This was first seen with the $S M M / G R S$ instrument in the flare of 3 June, 1982 (Forrest et al. 1985), and deduction of the accelerated ion distribution to high energies followed (Murphy et al. 1987). Alexander et al. (1994) paid similar attention to the $\pi^{0}$ 
spectral feature seen in two other SMM flares, using it to discuss in a parametric way the relationship between the accelerated proton distribution at $10-100 \mathrm{MeV}$, and at $>300 \mathrm{MeV}$. More recently, Dunphy et al. (1999) estimated the accelerated proton spectra for the flare of 11 June, 1991 using $C G R O / E G R E T$ observations of $\gamma$-ray emissions in the 1 to $140 \mathrm{MeV}$ range. In the delayed phase of the flare, energetic neutrons and pion decay radiation were clearly observed implying the hardening of the ion spectrum in this phase compared to the impulsive flare phase. Estimates of the photon spectra were respectively made from the ratio of the fluence of the delayed 2.2 MeV line to the prompt $\gamma$-ray lines in the 4-7 MeV range and from the ratio of the fluence of the $\pi^{0}$ decay component to the 4-7 MeV fluence. While in the delayed phase, the production of the lines and of the pion decay components was found to be consistent with a single power law ion spectrum, in the impulsive phase, in which only an upper limit of the fluence of the pion decay radiation was obtained, it was consistent with Bessel functions or with a power law spectra with a softening at about $100 \mathrm{MeV}$. Kocharov et al. (1998) studied the flare of 15 June, 1991 combining GAMMA-1 observations of pion radiation up to $1 \mathrm{GeV}$ with measurements from Comptel of neutrons and GRLs and investigated the behaviour of the energetic ion spectra in the 10-200 MeV range, around $300 \mathrm{MeV}$ and in the $\mathrm{GeV}$ range. They found that to reproduce all the observations the ion distribution should soften around $300 \mathrm{MeV}$ and harden again at $\mathrm{GeV}$ energies. This finding was later discussed, with a refined treatment of Comptel's neutron response, in Toner et al. (2001). Finally, Kocharov et al. (1994, 1996) combined GRANAT observations, for the flare of 24 May 1991, of the delayed 2.2 MeV line and of the pion decay radiation up to $100 \mathrm{MeV}$ with neutron observations from groundlevel neutron monitors. They also showed that to reproduce the 2.2 $\mathrm{MeV}$ and neutron observations the ion energetic spectrum should soften around $300 \mathrm{MeV}$.

In previous work (Murphy et al. 1987; Mandzhavidze \& Ramaty 1992) pion production by fast $\alpha$ 's was included assuming that protons and $\alpha$ 's have identical energy distributions (in energy/nucleon), and that the accelerated $\alpha / \mathrm{p}$ abundance ratio was similar to normal (e.g. Cameron) abundances. Recent results obtained from $\gamma$-ray line spectroscopy of flares (Share \& Murphy 1998; Mandzhavidze et al. 1997) have led to the conclusion that the accelerated $\alpha / \mathrm{p}$ ratio can be as large as 0.5 in some flares. Thus there is now a case for reconsidering the inclusion of pion production by fast $\alpha$ 's.

In this paper we re-examine the interpretation of the high-energy pion-related GR emission observed by PHEBUS/GRANAT in the flare of 24 May, 1990 (Talon et al. 1993; Debrunner et al. 1997). As described in Sect. 2, the continuum above $10 \mathrm{MeV}$ photon energy clearly shows the flattening around $70 \mathrm{MeV}$ characteristic of pion decay radiation at various stages of this flare. In Sect. 4, we combine the PHEBUS observations above $10 \mathrm{MeV}$ with calculations (described in Sect. 3) of the pion decay continuum (including $\alpha$-p contributions) to quantitatively constrain the accelerated ion energy distribution at energies above $300 \mathrm{MeV}$. Using these new estimates of the ion spectra above $300 \mathrm{MeV}$ and the additional information provided by the prompt $\gamma$-ray line observations we re-examine whether it is possible to yield a consistent picture of the ions accelerated in a large energy range in this event.

\section{Summary of observations of the 24 May, 1990 flare}

The large flare of 24 May 1990 (GOES X9.3, N36 W76) was well observed by the PHEBUS experiment aboard GRANAT in both the gamma ray line domain $(2.2 \mathrm{MeV}$ and 4-7 MeV range), and at energies above $10 \mathrm{MeV}$ (Talon et al. 1993). It also produced high-energy neutrons (above $200 \mathrm{MeV}$ ) detected by ground-level neutron monitors. The event is described in detail in Debrunner et al. (1992, 1993, 1997), Kocharov et al. (1994, 1996) and Torsti et al. (1996). The neutron observations as well as the relation between interacting and interplanetary particles were extensively discussed in Debrunner et al. (1992, 1993, 1997), Kocharov et al. (1994, 1996), Kovaltsov et al. (1994) and Torsti et al. (1996). In the present paper, we focuss on the quantitative analysis of the pion decay radiation. Below, we summarize the results of the work by Debrunner et al. (1997) which are relevant to the present analysis. The flare comprises an impulsive phase $(\simeq 2047: 50-2049: 24$ UT) followed by a long duration tail at high energies which was still going on at the end of PHEBUS observations ( $\simeq 2057$ UT). This long lasting emission is dominated by high-energy gamma-ray continuum and by $>500 \mathrm{MeV}$ neutrons. The present analysis is restricted to the time period 2047:50-2050:10 UT before the arrival of $>500 \mathrm{MeV}$ neutrons at GRANAT (Debrunner et al. 1997). Figure 1 shows the time profile of the PHEBUS count rates until $2051 \mathrm{UT}$ in the $10-24 \mathrm{MeV}, 60-75 \mathrm{MeV}$ and $75-$ $95 \mathrm{MeV}$ channels. The four time intervals (marked 1 to 4 ) where detailed spectral analysis is performed are also indicated. The second peak at about 2048:35 UT appearing only in the $60-75 \mathrm{MeV}$ and $75-95 \mathrm{MeV}$ channels, strongly suggests pronounced pion decay radiation at this time. Such radiation was observed throughout the latter part of the impulsive phase, and on into the decay phase of the flare.

De-excitation $\gamma$-ray line emission was also observed (Debrunner et al. 1997) and was used to estimate energetic proton numbers above $30 \mathrm{MeV}$. This last work was based on $2.2 \mathrm{MeV}$ and prompt $\gamma$-ray line time profiles corrected from instrumental effects such as dead time, pulse pile up and energy scale non linearities (see Debrunner et al. 1997 for a discussion of these corrected time profiles when compared to the previous ones published by Terekhov et al. 1993). The corrected integrated fluences for the gamma-ray line emission were found to be $\sim 1000$ and 960 photons $\mathrm{cm}^{-2}$ in, respectively, the neutron capture line at $2.2 \mathrm{MeV}$, and the 3.7-7.7 MeV band (Debrunner et al 1997). The $2.2 \mathrm{MeV}$ to the $3.7-7.7 \mathrm{MeV}$ fluence ratio from 2048:24 UT (start of the second $\gamma$-ray peak) to the end of the event is found to be $1.0 \pm 0.2$. Using the calculations of Hua \& Lingenfelter (1987) the spectral index of the power-law energy spectrum $\delta$ is $\simeq-2.5-3.0$ for a few $\mathrm{MeV} /$ nuc to a few $100 \mathrm{MeV} /$ nuc interacting protons. Debrunner et al. (1997) also estimated that $5 \times 10^{33}$ to $10^{34}$ protons above $30 \mathrm{MeV}$ were necessary to account for the observed $2.2 \mathrm{MeV}$ fluence for such a value of $\delta$. 


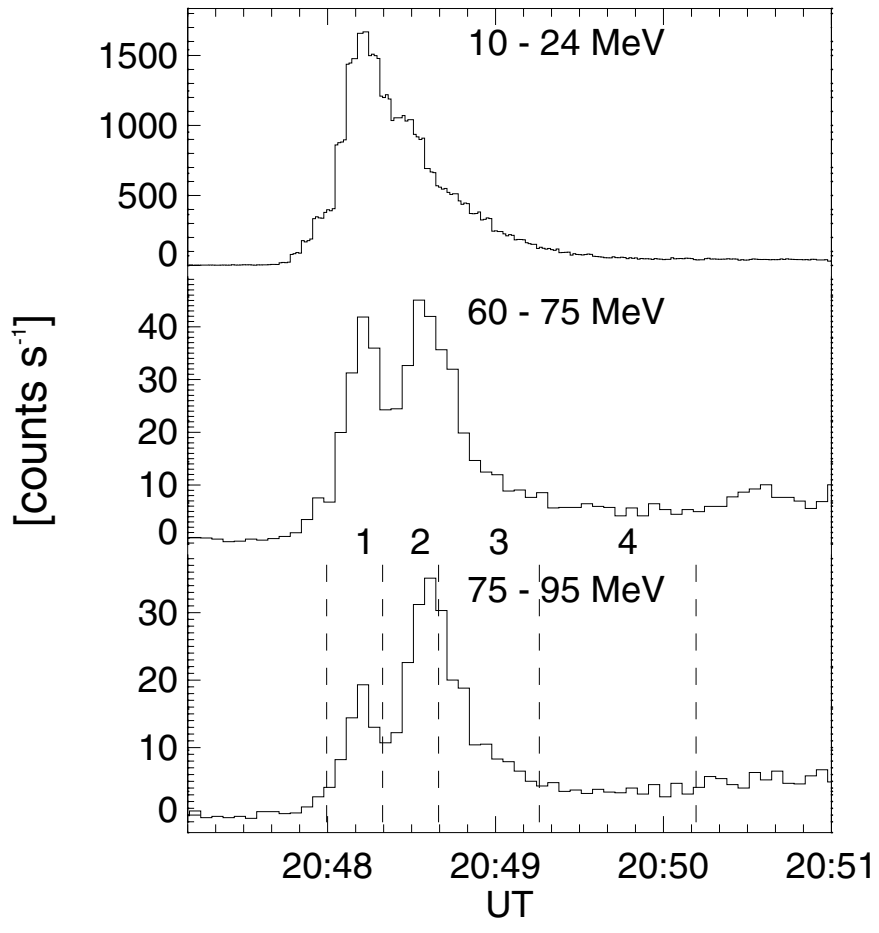

Fig. 1. Temporal development of the 24 May 1990 event, as viewed in 10-24, 60-75 and 75-95 MeV energy channels. The four time intervals used for spectral analysis (marked 1 to 4) are also indicated on the bottom curve.

The neutron spectrum deduced by Debrunner et al. (1997) is a power law energy spectrum with $s=-2.9 \pm 0.1$ in the first part of the flare (from 2048:18 UT to 2049:24 UT), and $s=-4.3 \pm 0.2$ in the last part of the flare (long duration tail) with a high energy cut-off of $\simeq 2 \mathrm{GeV}$. It was found that about $10^{34}$ protons above $30 \mathrm{MeV}$ with $\delta \simeq-2.5-3$ were necessary to account for the estimated neutron emissivity of $3.5 \times 10^{30} \mathrm{sr}^{-1}$ above $100 \mathrm{MeV}$. Such a number is in agreement with that obtained from the estimated values of the $\gamma$-ray line fluences. A high energy cut-off above $2 \mathrm{GeV}$ is also expected for the proton spectrum given the neutron high energy cut-off at $2 \mathrm{GeV}$ deduced from observations. Below we discuss these findings further, and their consistency with the observed pion decay radiation.

\section{Pion decay radiation}

\subsection{Calculation of pion decay $\gamma$-ray spectrum}

We calculate the pion decay $\gamma$-radiation resulting from assumed populations of accelerated protons and alphas, for detailed comparison with the PHEBUS data. For photon production via $\pi^{0}$ decay resulting from $\mathrm{p}-\mathrm{p}$ and $\mathrm{p}-\alpha$ interactions, we follow Alexander et al. (1994), who in turn mostly followed the approach of Dermer (1986a) and Murphy et al. (1987). In this work we also evaluate the contribution from $\alpha$-p interactions, noting that collisions of $\alpha$ 's with other $\alpha$ 's, and heavier nuclei, will add only a few percent to the total photon yield (Murphy et al. 1987). Pion production in $\alpha$-p reactions is calculated following Dermer (1986b), assuming that pion production takes place primarily via one proton of the incident $\alpha$ particle. With this assumption, $\mathrm{p}-\alpha$ and $\alpha$-p reactions produce the same photon yield, at the same test particle energy (in $\mathrm{MeV}$ per nucleon). We also extend the work of Alexander et al. (1994) by including the continuum resulting from bremsstrahlung and annihilation in flight of $\pi^{+}$decay positrons (the contribution of $\pi^{-}$decay electrons to the total gamma-ray fluence is at the $1 \%$ level Murphy et al. 1987 - so we neglect it). The production rate and energy distribution of $\pi^{+}$is calculated similarly to Alexander et al. using measured cross-sections for $\pi^{+}$production, and isobar and scaling models for the $\pi^{+}$energy distribution (Dermer 1986b). We note that the isobar and scaling approach (Dermer 1986a, 1986b) used here has been validated using Monte Carlo codes incorporating detailed models of hadronic interactions (Mori 1997). The distribution of $\mathrm{e}^{+}$then follows as in Dermer (1986b), and the analytical expressions of Murphy et al. (1987) are used for the consequent bremsstrahlung and annihilationin-flight photon yields. These include the possibility of significant synchrotron losses, which may shorten the radiatively effective lifetime of the positrons and depress the photon yield below its ideal, "collision-dominated thick target" value. For this purpose we assume that single values of ambient density $n$ and magnetic field strength $B$ characterise the whole source. For a given injected proton energy distribution, the photon yield per injected proton then depends on the relative magnitudes of synchrotron and collisional losses, determined by the ratio $B^{2} / n$.

Let $F(E) \mathrm{d} E$ be the total (i.e. time-integrated) number of protons injected into the source with kinetic energies between $E$ and $E+\mathrm{d} E$. We assume that $F(E)$ has power-law form in the energy range of interest, with an upper cutoff $E_{\max }$ :

$F(E)=A E^{-\delta} H\left(E_{\max }-E\right)$

where $H$ is the Heaviside step function. We pick the normalisation constant $A$ so that the number of protons above $30 \mathrm{MeV}$ equals 1 . Proton energy thresholds for pion production are in the region of $300 \mathrm{MeV}$, but we adopt this normalisation for comparison with previous work (in particular, Murphy et al. 1987), and with inferences from de-excitation lines.

Just a couple of other features of these calculations deserve special mention. First, we continue with the practice (Murphy et al. 1987; Alexander et al. 1994) of degrading the injected distribution by the probability of survival against elastic and inelastic nuclear collisions. Mandzhavidze \& Ramaty (1992) pointed out that this underestimates the eventual photon yield, because many elastic collisions result in knock-on particles of comparable energies to their progenitors. They compensated for this by neglecting elastic collisions altogether. In fact a complete treatment of knock-on particles in a thick target appears possible. Details will be presented elsewhere; we continue with previous practice as an interim measure, noting that this underestimates the photon yield by a few 10 s of $\%$ (Murphy et al. 1987). Also, particle angular distributions in the source are assumed isotropic; alternatively, the calculations may be regarded as averaged over viewing angle. 


\subsection{Pion decay $\gamma$-ray spectrum expected for different energetic proton and $\alpha$ particle spectra}

In Figs. 2 and 3 we present examples of the results of these calculations, for bombarding protons and $\alpha$ particles respectively. Photon yield at the Sun per MeV per proton are computed and these numbers must be divided by $4 \pi R^{2}$ where $R$ is $1 \mathrm{AU}$ and multiplied by the total number of protons between $30 \mathrm{MeV}$ and an upper cut-off to give a flux per $\mathrm{MeV}$ per second per $\mathrm{cm}^{2}$ at Earth. For interpretation of observations it is important to bear in mind the overall form of the pion decay photon spectrum: the flat, broad "bump" feature centered on $67 \mathrm{MeV}$ resulting from $\pi^{0}$ decay (Stecker 1970), superimposed on the hard continuum produced by $\pi^{+}$decay products. This continuum drops to zero at a maximum determined simply by kinematics and the maximum energy of $\mathrm{e}^{+}$produced by the maximum energy of proton injected (Dermer 1986b). The exact form and intensity of this component also depend on the value of the magnetic field: increasing the magnetic field increases the synchrotron loss rate of secondary positrons and thus reduces the contribution of the charged pions to the total radiation spectrum. While Fig. 2 shows results from $\mathrm{p}-\mathrm{p}$ and $\mathrm{p}-\alpha$ reactions (with an ambient helium abundance of $7 \%$, i.e. Cameron abundances), Fig. 3 shows results for the case when only accelerated $\alpha$ 's are present. We see that the relative importance of the $\pi^{0}$ and $\pi^{+}$spectral components not only depends on $B^{2} / n$, but also on the nature of the bombarding particles ( $\alpha$ particles or protons). This results from the different behaviours with incident energies (in $\mathrm{MeV} /$ nuc) of the $\mathrm{p}$-p and $\alpha$-p cross sections (see e.g. Figs. 3 and 4 of Murphy et al. 1987). The bump or flattening of the total radiation due to $\alpha$ is thus more pronounced. As we shall see below, existing observations probably do not allow diagnostic exploitation of these dependences.

In the photon energy range appropriate here, an increase in $\delta$ with $E_{\max }$ and the number of ions above $300 \mathrm{MeV}$ fixed results primarily in more photons being produced per accelerated particle; effects on the spectral shape are secondary. An increase in $E_{\max }$ with fixed $\delta$ and number of ions above $300 \mathrm{MeV}$, on the other hand, both broadens the neutral pion spectral feature, hardens the spectrum and increases the photon yield per accelerated ion. Moreover, the spectrum displays a sharp cutoff at a maximum photon energy determined by $E_{\max }$. If observed, this upper cutoff would eliminate one degree of uncertainty in the ion distribution in a decisive way. We shall see below that PHEBUS did not probe high enough photon energy range to infer strong constraints on the exact shape of the incident proton spectrum from the observations of pion decay radiation.

\section{High energy protons deduced from high-energy radiation above $10 \mathrm{MeV}$}

A detailed spectral analysis has been performed on the highenergy $(\geq 10 \mathrm{MeV})$ part of the $\gamma$-ray spectrum in the impulsive flare phase and the very start of the long duration tail, before the arrival of solar neutrons on the PHEBUS detector. Background subtracted count-rate spectra accumulated during the four time intervals marked 1 to 4 in Fig. 1 have been considered. They correspond resp. to the first peak (1), the rise (2) and decay
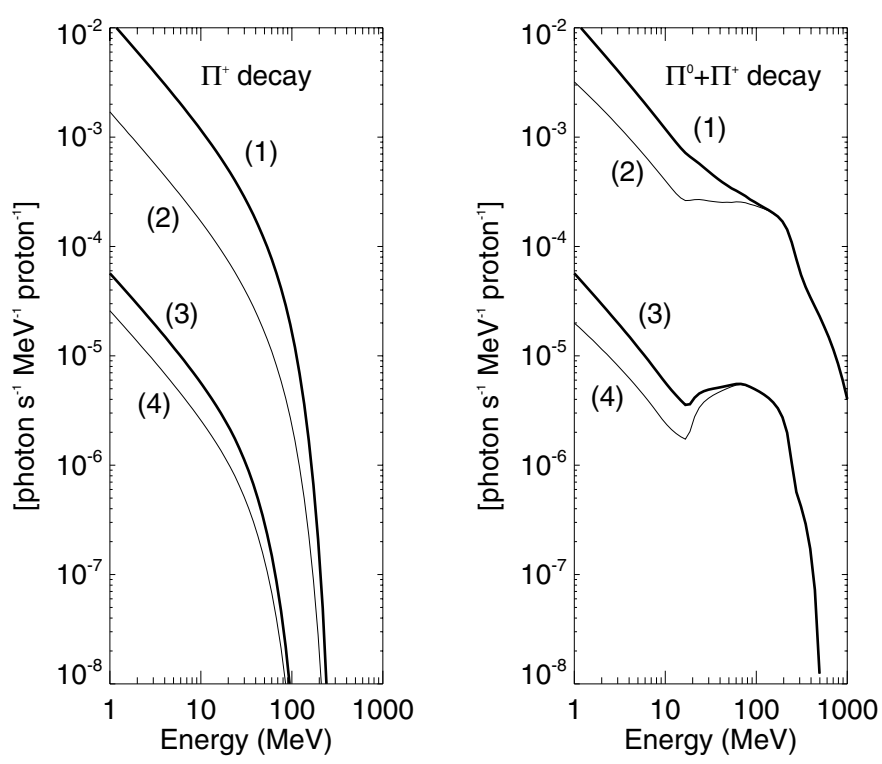

Fig. 2. Left: photon yield at the Sun from charged pion decay for the following parameters: $\delta=2, B=1 \mathrm{G}, E_{\max }=3 \mathrm{GeV}$ (1); and $E_{\max }=750 \mathrm{MeV}(3) ; \delta=4, B=1 \mathrm{G}, E_{\max }=3 \mathrm{GeV}$ (2); and $E_{\max }=750 \mathrm{MeV}$ (4). Right: total photon yield at the Sun from neutral and charged pion decay for the following parameters: $\delta=2, B=1 \mathrm{G}$, $E_{\max }=3 \mathrm{GeV}(1)$; and $E_{\max }=750 \mathrm{MeV}(3) ; \delta=2, B=1000 \mathrm{G}$, $E_{\max }=3 \mathrm{GeV}(2)$; and $E_{\max }=750 \mathrm{MeV}$ (4). All curves are normalised to one proton above $30 \mathrm{MeV}$.

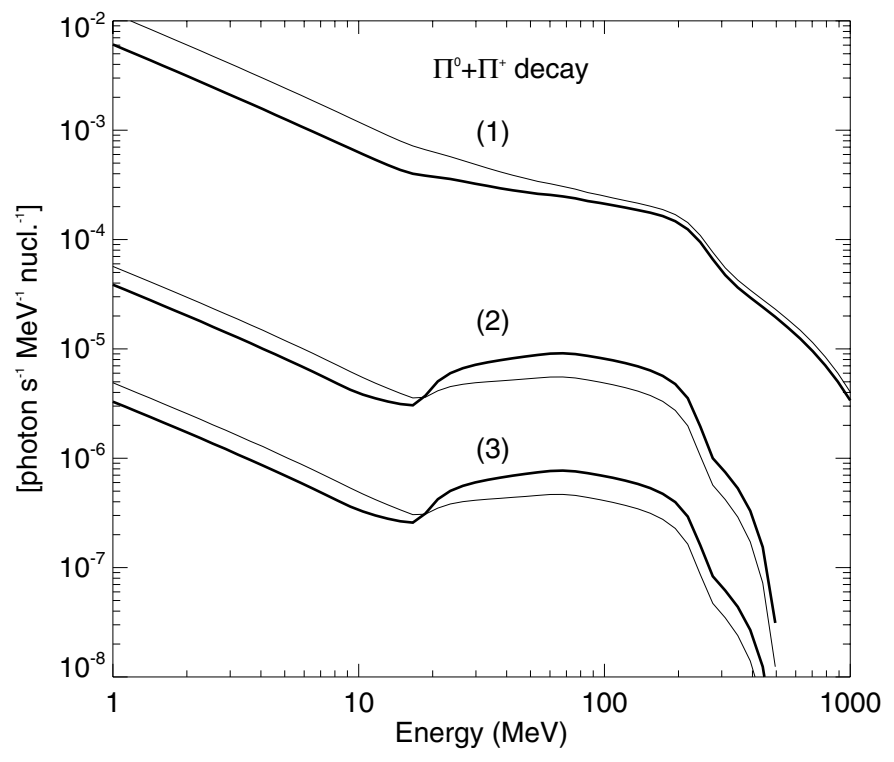

Fig. 3. Thick lines: photon yield at the Sun from neutral and charged pion decay produced by energetic $\alpha$ particles for the following parameters: $\delta=2$ (energy spectrum in $\mathrm{MeV} /$ nuc), $B=1 \mathrm{G}, E_{\max }=$ $3 \mathrm{GeV}(1)$; and $E_{\max }=750 \mathrm{MeV}(2) ; \delta=3, B=1 \mathrm{G}, E_{\max }=750 \mathrm{MeV}$ (3). Thin lines: similar plots for the radiation arising from energetic protons with similar spectra in $\mathrm{MeV}$. All curves are normalised for one particle above $30 \mathrm{MeV} /$ nucl.

(3) of the second peak and the beginning of the long duration tail (4) in the 75-95 MeV band. For each of the four spectra, different trial spectra of incident photons are considered and convolved with the detector response function. The count spectra obtained are compared to the observed ones and a $\chi^{2}$ 

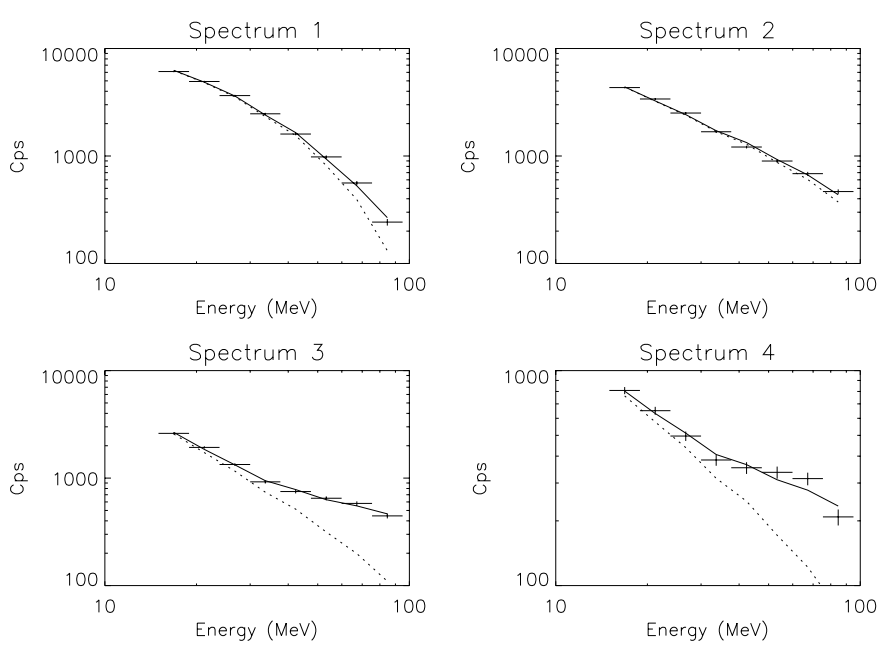

Fig. 4. Background subtracted count spectra observed by PHEBUS/GRANAT during time intervals 1 to 4 in the $10 \mathrm{MeV}-$ $100 \mathrm{MeV}$ energy range. The full line indicates one of the best fits found above $10 \mathrm{MeV}$ for an electron bremstrahlung component and a pion contribution. The dotted line shows the only contribution of the electron bremstrahlung component. See text for details.

minimization algorithm is used to determine the free parameters providing the best fit to the observed count spectrum. In the first instance, spectral fitting is restricted to this high energy part and no pion decay radiation from $\alpha$ 's is included. The trial spectra consist of:

- a bremsstrahlung continuum produced by relativistic electrons represented by a single power law with or without an exponential high energy cut-off $\left(A_{\mathrm{e}} E^{-\gamma}\right.$ or $\left.A_{\mathrm{e}} E^{-\gamma} \mathrm{e}^{-E / E_{\text {roll }}}\right)$

- a total (neutral and charged) pion decay $\gamma$-ray spectrum determined by the proton spectral index $\delta$, the proton highenergy cut-off $E_{\max }$, the ratio $B^{2} / n$ of extrinsic parameters and scaled by the total number $N_{p>30 \mathrm{MeV}}$ of energetic protons between $30 \mathrm{MeV}$ and $E_{\max }$ (which is the only free parameter left).

For each analysed spectrum, different trials were performed with different shapes of the continuum and the contribution (or not) of pion radiation. The free parameters are then $A_{\mathrm{e}}$ and $\gamma$ ( $E_{\text {roll }}$ if a high energy cut-off is considered) and $N_{p>30} \mathrm{MeV}$ when the possibility of a pion contribution is included. The number of energy channels above $10 \mathrm{MeV}$ is too small to determine a unique set of parameters. Indeed, similar values of $\chi^{2}$ are obtained for different shapes of the proton spectrum and of the electron high energy continuum. It is also found that the number of protons deduced from pion decay radiation is largely independant of $B^{2} / n$, i.e. on the efficiency of gyrosynchrotron losses for pion decay positrons. This shows that the contribution of charged pion decay radiation plays no significant role in determining the fit of the present spectra, since this contribution is not dominant in the spectral domain fitted here (i.e. essentially above $20 \mathrm{MeV}$ ), certainly not to a degree that would allow it to be disentangled from primary electron continuum. We note this retrospectively validates the concentration on the $\pi^{0}$ spectral feature, and the neglect of the charged pion continuum, in the work of Alexander et al. (1994).
Table 1. Combination of likely parameters deduced for the countrate spectrum above $10 \mathrm{MeV}$ accumulated from 20:47:59:648 UT to 20:48:19:648 UT (first high energy peak, interval 1).

\begin{tabular}{|c|c|c|}
\hline \multicolumn{3}{|c|}{ e bremsstrahlung radiation } \\
\hline$A_{\mathrm{e}}$ & $\gamma$ & $E_{\text {roll }}(\mathrm{MeV})$ \\
\hline \multicolumn{3}{|c|}{ if no pion contribution } \\
\hline $4 \times 10^{5}$ & 1.7 & 42 \\
\hline \multicolumn{3}{|c|}{ if pion contribution } \\
\hline $2 \times 10^{5}$ & 1.4 & 28 \\
\hline \multicolumn{3}{|c|}{ proton spectrum } \\
\hline \multicolumn{3}{|c|}{$\delta=2$} \\
\hline$E_{\max }(\mathrm{MeV})$ & $N_{p>30 \mathrm{MeV}}$ & $N_{p>300 \mathrm{MeV}}$ \\
\hline 750 & $3 \times 10^{31}$ & $2 \times 10^{30}$ \\
\hline 1000 & $4 \times 10^{30}$ & $3 \times 10^{29}$ \\
\hline 2000 & $1 \times 10^{30}$ & $8 \times 10^{28}$ \\
\hline 3000 & $8 \times 10^{29}$ & $7 \times 10^{28}$ \\
\hline \multicolumn{3}{|c|}{$\delta=3$} \\
\hline$E_{\max }(\mathrm{MeV})$ & $N_{p>30 \mathrm{MeV}}$ & $N_{p>300 \mathrm{MeV}}$ \\
\hline 750 & $4 \times 10^{32}$ & $3 \times 10^{30}$ \\
\hline 1000 & $7 \times 10^{31}$ & $6 \times 10^{29}$ \\
\hline 2000 & $2 \times 10^{31}$ & $2 \times 10^{29}$ \\
\hline 3000 & $2 \times 10^{31}$ & $2 \times 10^{29}$ \\
\hline \multicolumn{3}{|c|}{$\delta=4$} \\
\hline$E_{\max }(\mathrm{MeV})$ & $N_{p>30 \mathrm{MeV}}$ & $N_{p>300 \mathrm{MeV}}$ \\
\hline 750 & $5 \times 10^{33}$ & $5 \times 10^{30}$ \\
\hline 1000 & $1 \times 10^{33}$ & $1 \times 10^{30}$ \\
\hline 2000 & $5 \times 10^{32}$ & $5 \times 10^{29}$ \\
\hline 3000 & $5 \times 10^{32}$ & $5 \times 10^{29}$ \\
\hline
\end{tabular}

Tables 1 to 4 summarize the results of likely fits obtained leading to similar values of $\chi_{\mathrm{r}}^{2}$ for the different spectra. Figure 4 shows the count-rate spectra accumulated over the four time intervals together with examples of likely spectral fits (the chosen parameters are among the ones leading to the smallest values of $\chi_{\mathrm{r}}^{2}$ ). The first analysed spectrum (first high energy peak) can be as well fitted with or without pion contribution to the photon spectrum (reduced $\chi_{\mathrm{r}}^{2}$ of 2.5 instead of 3.4), but an exponential high energy cut-off of the bremsstrahlung continuum is needed in both cases. Table 1 thus only indicates an upper limit of the number of protons which may contribute to the observed photon spectrum, once the spectral index of protons is fixed.

For spectra 2 to 4 , the inclusion of pion decay radiation leads to increasingly better fits, in good consistency with the previous qualitative analysis of Debrunner et al. (1997). However, while for spectrum 2 the inclusion of pion decay radiation only reduces $\chi_{\mathrm{r}}^{2}$ from 6 to 4 , for spectra 3 and 4 the inclusion of pion decay radiation considerably improves the fit $\left(\chi_{\mathrm{r}}^{2}\right.$ of 2 instead of 20). Thus the relative contribution of pion decay radiation increases with time with respect to the electron component. The photon spectra are, however, not measured to high enough energies to unambiguously determine the primary proton energy distributions. Indeed, spectral fits leading to similar values of $\chi_{\mathrm{r}}^{2}$ are found for different shapes of the primary proton energy distributions. Only acceptable regions of the proton 
Table 2. Combination of likely parameters deduced for the countrate spectrum above $10 \mathrm{MeV}$ accumulated from 20:48:19:648 UT to 20:48:39:648 UT (second high energy peak, interval 2).

\begin{tabular}{|c|c|c|}
\hline \multicolumn{3}{|c|}{ e bremsstrahlung radiation } \\
\hline$A_{\mathrm{e}}$ & $\gamma$ & $E_{\text {roll }}(\mathrm{MeV})$ \\
\hline \multicolumn{3}{|c|}{ if no pion contribution } \\
\hline $1 \times 10^{6}$ & 2.4 & \\
\hline \multicolumn{3}{|c|}{ if pion contribution } \\
\hline $1 \times 10^{6}$ & 2.4 & \\
\hline $3 \times 10^{5}$ & 1.7 & 42 \\
\hline \multicolumn{3}{|c|}{ proton spectrum } \\
\hline \multicolumn{3}{|c|}{$\delta=2$} \\
\hline$E_{\max }(\mathrm{MeV})$ & $N_{p>30 \mathrm{MeV}}$ & $N_{p>300 \mathrm{MeV}}$ \\
\hline 750 & $3 \times 10^{31}-9 \times 10^{31}$ & $2 \times 10^{30}-5 \times 10^{30}$ \\
\hline 1000 & $5 \times 10^{30}-1 \times 10^{31}$ & $4 \times 10^{29}-7 \times 10^{29}$ \\
\hline 2000 & $1 \times 10^{30} 3 \times 10^{30}$ & $9 \times 10^{28} 3 \times 10^{29}$ \\
\hline 3000 & $7 \times 10^{29}-2 \times 10^{30}$ & $6 \times 10^{28}-2 \times 10^{29}$ \\
\hline \multicolumn{3}{|c|}{$\delta=3$} \\
\hline$E_{\max }(\mathrm{MeV})$ & $N_{p>30 \mathrm{MeV}}$ & $N_{p>300 \mathrm{MeV}}$ \\
\hline 750 & $4 \times 10^{32}-1 \times 10^{33}$ & $3 \times 10^{30}-8 \times 10^{30}$ \\
\hline 1000 & $7 \times 10^{31}-2 \times 10^{32}$ & $6 \times 10^{29}-2 \times 10^{30}$ \\
\hline 2000 & $2 \times 10^{31}-5 \times 10^{31}$ & $2 \times 10^{29}-5 \times 10^{29}$ \\
\hline 3000 & $2 \times 10^{31}-4 \times 10^{31}$ & $2 \times 10^{29}-4 \times 10^{29}$ \\
\hline \multicolumn{3}{|c|}{$\delta=4$} \\
\hline$E_{\max }(\mathrm{MeV})$ & $N_{p>30 \mathrm{MeV}}$ & $N_{p>300 \mathrm{MeV}}$ \\
\hline 750 & $5 \times 10^{33}-1 \times 10^{34}$ & $5 \times 10^{30}-9 \times 10^{30}$ \\
\hline 1000 & $1 \times 10^{33}-3 \times 10^{33}$ & $1 \times 10^{30}-3 \times 10^{30}$ \\
\hline 2000 & $6 \times 10^{32}-1 \times 10^{33}$ & $6 \times 10^{29}-1 \times 10^{30}$ \\
\hline 3000 & $5 \times 10^{32}-1 \times 10^{33}$ & $5 \times 10^{29}-1 \times 10^{30}$ \\
\hline
\end{tabular}

distribution parameter space can thus be delineated: basically the number of protons above $30 \mathrm{MeV}$ (and above $300 \mathrm{MeV}$ ) is fixed once the maximum energy and energy spectral index are assumed. Finally, although inclusion of pion decay radiation lead to better fits of spectra 2 to 4 , the deduced amount of pion radiation is, due to the limited observational spectral constraints at high energies, not completely independant on the shape of the high energy electron bremsstrahlung. This is illustrated by the results of two different trials performed for spectra 2 to 4 . In the first one spectral fitting of both electron and ion components is performed (first line of the results of electron bremsstrahlung component with pion contribution and first value of the number of protons). In the second one, the shape of electron bremsstrahlung component (but not the amplitude) is kept constant as it was in the first spectrum and only the fit of the pion component is performed (second line of the results of electron bremsstrahlung component and second value of the number of protons). This assumes that the electron component keeps a similar hardness during the whole event. As it is clear from Tables 3 and 4 , the shape of the electron continuum is not as important in determining the amount of pion radiation and thus the number of protons for spectra 3 and 4 as it is for spectrum 2. This is consistent with the fact that the bump due to a neutral pion component is clearly seen in the count-rate
Table 3. Combination of likely parameters deduced for the countrate spectrum above $10 \mathrm{MeV}$ accumulated from 20:48:39:648 UT to 20:49:15:648 UT (decay phase of the second high energy peak, interval 3).

\begin{tabular}{|c|c|c|}
\hline \multicolumn{3}{|c|}{ e bremsstrahlung radiation } \\
\hline$A_{\mathrm{e}}$ & $\gamma$ & $E_{\text {roll }}(\mathrm{MeV})$ \\
\hline \multicolumn{3}{|c|}{ if no pion contribution } \\
\hline $4 \times 10^{5}$ & 2.2 & \\
\hline \multicolumn{3}{|c|}{ if pion contribution } \\
\hline $2 \times 10^{6}$ & 2.8 & \\
\hline $1 \times 10^{5}$ & 1.7 & 42 \\
\hline \multicolumn{3}{|c|}{ proton spectrum } \\
\hline \multicolumn{3}{|c|}{$\delta=2$} \\
\hline$E_{\max }(\mathrm{MeV})$ & $N_{p>30 \mathrm{MeV}}$ & $N_{p>300 \mathrm{MeV}}$ \\
\hline 750 & $1 \times 10^{32}-1 \times 10^{32}$ & $8 \times 10^{30}-8 \times 10^{30}$ \\
\hline 1000 & $1 \times 10^{31}-1 \times 10^{31}$ & $1 \times 10^{30}-1 \times 10^{30}$ \\
\hline 2000 & $3 \times 10^{30}-3 \times 10^{30}$ & $3 \times 10^{29}-3 \times 10^{29}$ \\
\hline 3000 & $2 \times 10^{30}-2 \times 10^{30}$ & $2 \times 10^{29}-2 \times 10^{29}$ \\
\hline \multicolumn{3}{|c|}{$\delta=3$} \\
\hline$E_{\max }(\mathrm{MeV})$ & $N_{p>30 \mathrm{MeV}}$ & $N_{p>300 \mathrm{MeV}}$ \\
\hline 750 & $1 \times 10^{33}-1 \times 10^{33}$ & $1 \times 10^{31}-1 \times 10^{31}$ \\
\hline 1000 & $2 \times 10^{32}-2 \times 10^{32}$ & $2 \times 10^{30}-2 \times 10^{30}$ \\
\hline 2000 & $7 \times 10^{31}-6 \times 10^{31}$ & $7 \times 10^{29}-6 \times 10^{29}$ \\
\hline 3000 & $5 \times 10^{31}-5 \times 10^{31}$ & $5 \times 10^{29}-5 \times 10^{29}$ \\
\hline \multicolumn{3}{|c|}{$\delta=4$} \\
\hline$E_{\max }(\mathrm{MeV})$ & $N_{p>30 \mathrm{MeV}}$ & $N_{p>300 \mathrm{MeV}}$ \\
\hline 750 & $2 \times 10^{34}-2 \times 10^{34}$ & $2 \times 10^{31}-2 \times 10^{31}$ \\
\hline 1000 & $4 \times 10^{33}-4 \times 10^{33}$ & $4 \times 10^{30}-4 \times 10^{30}$ \\
\hline 2000 & $2 \times 10^{33}-2 \times 10^{33}$ & $2 \times 10^{30}-2 \times 10^{30}$ \\
\hline 3000 & $2 \times 10^{33}-1 \times 10^{33}$ & $2 \times 10^{30}-1 \times 10^{30}$ \\
\hline
\end{tabular}

spectra 3 and 4, and that such a bump cannot be mimicked by bremsstrahlung. On the other hand, for spectrum 2, the amount of pion radiation and thus the number of protons are very dependent on the shape of the electron component varying on one order of magnitude. This simply reflects the fact that for this spectrum the bump due to pion decay radiation is not clearly evidenced.

\section{Consistency of the deduced spectra in the whole energy range?}

\subsection{Electron bremsstrahlung radiation above $300 \mathrm{keV}$ and above $10 \mathrm{MeV}$}

As shown in the previous section, the shape of the electron bremsstrahlung above $10 \mathrm{MeV}$ and the amount of high energy radiation due to pion decay are not completely independent and several combinations leading to equally likely fits can be found. The search for consistency between the electron bremsstrahlung spectrum at low and high energies could provide additional information to discriminate between several combinations. However, as already pointed above and in Debrunner et al. (1997), the 1990 May 24 event is a very 
Table 4. Combination of likely parameters deduced for the countrate spectrum above $10 \mathrm{MeV}$ accumulated from 20:49:15:648 UT to 20:50:11:648 UT (beginning of the long-duration tail, interval 4).

\begin{tabular}{|c|c|c|}
\hline \multicolumn{3}{|c|}{ e bremsstrahlung radiation } \\
\hline$A_{\mathrm{e}}$ & $\gamma$ & $E_{\text {roll }}(\mathrm{MeV})$ \\
\hline \multicolumn{3}{|c|}{ if no pion contribution } \\
\hline $1 \times 10^{5}$ & 2.1 & \\
\hline \multicolumn{3}{|c|}{ if pion contribution } \\
\hline $2 \times 10^{5}$ & 2.3 & \\
\hline $4 \times 10^{4}$ & 1.7 & 42 \\
\hline \multicolumn{3}{|c|}{ proton spectrum } \\
\hline \multicolumn{3}{|c|}{$\delta=2$} \\
\hline$E_{\max }(\mathrm{MeV})$ & $N_{p>30 \mathrm{MeV}}$ & $N_{p>300 \mathrm{MeV}}$ \\
\hline 750 & $5 \times 10^{31}-8 \times 10^{31}$ & $5 \times 10^{30}-7 \times 10^{30}$ \\
\hline 1000 & $6 \times 10^{30}-8 \times 10^{30}$ & $4 \times 10^{29}-6 \times 10^{29}$ \\
\hline 2000 & $2 \times 10^{30}-2 \times 10^{30}$ & $2 \times 10^{29}-2 \times 10^{29}$ \\
\hline 3000 & $1 \times 10^{30}-1 \times 10^{30}$ & $1 \times 10^{29}-1 \times 10^{29}$ \\
\hline \multicolumn{3}{|c|}{$\delta=3$} \\
\hline$E_{\max }(\mathrm{MeV})$ & $N_{p>30 \mathrm{MeV}}$ & $N_{p>300 \mathrm{MeV}}$ \\
\hline 750 & $6 \times 10^{32}-8 \times 10^{32}$ & $6 \times 10^{30}-8 \times 10^{30}$ \\
\hline 1000 & $1 \times 10^{32}-1 \times 10^{32}$ & $1 \times 10^{30}-1 \times 10^{30}$ \\
\hline 2000 & $3 \times 10^{31}-4 \times 10^{31}$ & $3 \times 10^{29}-4 \times 10^{29}$ \\
\hline 3000 & $3 \times 10^{31}-3 \times 10^{31}$ & $3 \times 10^{29}-3 \times 10^{29}$ \\
\hline \multicolumn{3}{|c|}{$\delta=4$} \\
\hline$E_{\max }(\mathrm{MeV})$ & $N_{p>30 \mathrm{MeV}}$ & $N_{p>300 \mathrm{MeV}}$ \\
\hline 750 & $8 \times 10^{33}-1 \times 10^{34}$ & $8 \times 10^{30}-1 \times 10^{31}$ \\
\hline 1000 & $2 \times 10^{33}-2 \times 10^{33}$ & $2 \times 10^{30}-3 \times 10^{30}$ \\
\hline 2000 & $9 \times 10^{32}-1 \times 10^{33}$ & $9 \times 10^{29}-1 \times 10^{30}$ \\
\hline 3000 & $8 \times 10^{32}-9 \times 10^{32}$ & $8 \times 10^{29}-9 \times 10^{29}$ \\
\hline
\end{tabular}

intense solar flare, and instrumental effects such as dead time and energy scale non linearities greatly complicate the spectral analysis especially at low energies. Therefore, spectral analysis in the low energy part of the spectrum (around a few hundred of $\mathrm{keV}$ ) must be taken with care during most of the event when the low energy count rate is very high. In the following, we analyse only the spectrum obtained during time interval 4 (i.e. at the start of the long duration tail) since at that time the count rate at low energies has largely diminished.

The complete spectrum observed by PHEBUS from $300 \mathrm{keV}$ to $100 \mathrm{MeV}$ during time interval 4 is shown in Fig. 5 . We investigate here whether the complete spectrum observed from $\simeq 300 \mathrm{keV}$ to $\simeq 1 \mathrm{MeV}$ and above $10 \mathrm{MeV}$ (i.e. outside the energy range where $\gamma$-ray line dominates) can be consistently reproduced with the same electron bremsstrahlung spectrum below $1 \mathrm{MeV}$ and above $10 \mathrm{MeV}$ and a pion decay component. This is a simplified approach for two reasons. First, hardening of the bremsstrahlung photon spectra above a few hundred keV has been reported in many flares (e.g. Dennis 1988) and even for a strong $\gamma$-ray line event (Barat et al. 1994). However, for this last event the hardening was observed above 200 to $400 \mathrm{keV}$. If such a hardening exists in the flare under study it should not modify the present conclusions given the fitted energy range. Second, the nuclear line component as well as the bremsstrahlung component in the 1 to $10 \mathrm{MeV}$ range will

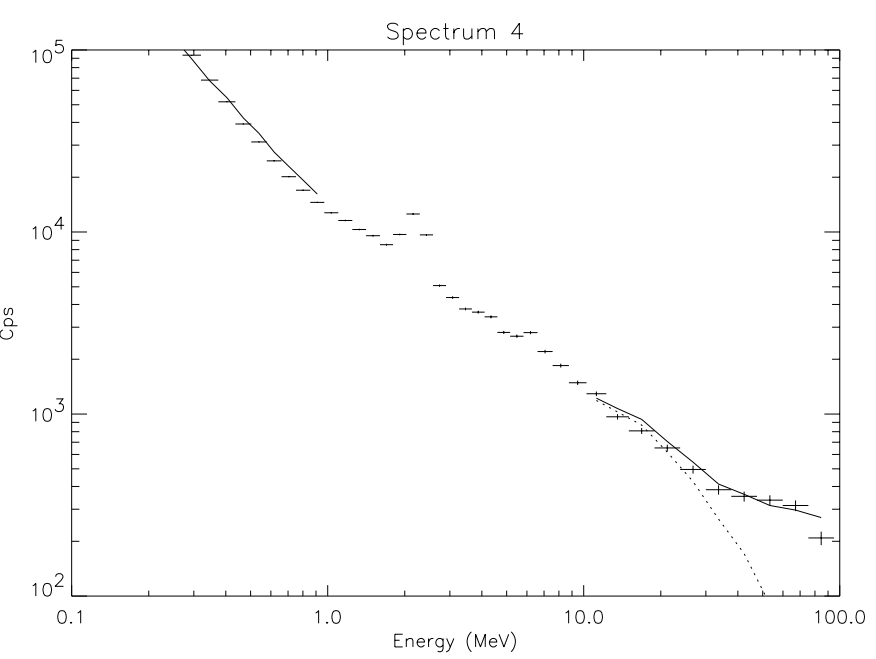

Fig. 5. Background subtracted count spectrum observed by PHEBUS/GRANAT during time interval 4 in the $300 \mathrm{keV}-100 \mathrm{MeV}$ energy range. The full line indicates one of the best fit found in the $300 \mathrm{keV}-1 \mathrm{MeV}$ range and above $10 \mathrm{MeV}$ for a single electron bremstrahlung component and a pion contribution. The dotted line shows the only contribution of the electron bremstrahlung component. The fit is obtained with the following parameters: $A_{\mathrm{e}}=1 \times 10^{5}, \gamma=2.0$, $E_{\text {roll }}=40 \mathrm{MeV}, N_{p>30 \mathrm{MeV}}=8 \times 10^{31}$ for $\delta=2$ and $E_{\max }=750 \mathrm{MeV}$.

also produce a significant number of counts below $1 \mathrm{MeV}$. This should result in a slightly steeper bremsstrahlung component below $1 \mathrm{MeV}$ than the one discussed here. Figure 5 shows however that a relatively reasonable fit can be obtained in the tail of the event with a single electron bremsstrahlung component and a pion radiation component, for the energy ranges going from $300 \mathrm{keV}$ to $1 \mathrm{MeV}$ and above $10 \mathrm{MeV}$. The nuclear line component discussed in Debrunner et al. (1997) clearly appears as an excess above the fit. The trial spectrum is however too simple to give an acceptable fit to the spectrum in these widely different energy ranges, since the reduced $\chi_{\mathrm{r}}^{2}$ of the fitted energy channels above $300 \mathrm{keV}$ is around 60 with a strong contribution of the low energy part of the spectrum.

\subsection{Proton spectra and numbers from pion decay radiation and $\gamma$-ray line emission}

The count rate spectrum of Fig. 5 shows in the 1-8 MeV energy range the nuclear line emission produced by interacting protons above a few MeV. This emission was analysed in Debrunner et al. (1997). In principle, line emission can be used in different ways to estimate the spectral index $\delta$ of $\gamma$-ray line producing protons. However, the energy resolution of BGO scintillators and the limited number of energy channels do not allow detailed analysis of the nuclear line spectra. Kocharov et al. (1994) and Debrunner et al. (1997) obtained $\delta$ by using the ratio of the $2.2 \mathrm{MeV}$ to the 3.7-7.6 MeV energy band, but this method can lead to uncertainties as large as $\Delta \delta \simeq 1.5$ (Ramaty et al. 1996) due to uncertainties in e.g. the ratio of accelerated $\alpha$ to accelerated protons. Furthermore, the values of the fluences used in Kocharov et al. (1994) were not corrected from all the instrumental effects discussed above. Table 5 gives the excess fluence $\Phi_{4-7}$ in the $3.7-7.6 \mathrm{MeV}$ range deduced by 
Table 5. 4-7 MeV nuclear line fluence deduced for each spectrum. Numbers of protons above $30 \mathrm{MeV}$ deduced for different values of the proton spectral index.

\begin{tabular}{lccc}
\hline \hline spectrum & $\Phi_{4-7}\left(\mathrm{ph} \mathrm{cm}^{-2}\right)$ & $\delta$ & $N_{p>30 \mathrm{MeV}}$ \\
\hline 1 & 250 & 2 & $8 \times 10^{32}$ \\
& & 3 & $10^{33}$ \\
& & 4 & $5 \times 10^{32}$ \\
\hline 2 & 330 & 2 & $1 \times 10^{33}$ \\
& & 3 & $1 \times 10^{33}$ \\
& & 4 & $7 \times 10^{32}$ \\
\hline 3 & 310 & 2 & $1 \times 10^{33}$ \\
& & 3 & $1 \times 10^{33}$ \\
& & 4 & $6 \times 10^{32}$ \\
\hline 4 & 180 & 2 & $6 \times 10^{32}$ \\
& & 3 & $7 \times 10^{32}$ \\
& & 4 & $4 \times 10^{32}$ \\
\hline
\end{tabular}

using the time evolution of the 3.7-7.6 MeV line fluence shown in Debrunner et al. (1997). The number of $\gamma$-ray line producing protons above $30 \mathrm{MeV}$ as a function of adopted spectral index is then deduced using the 4 to $7 \mathrm{MeV}$ yields from Murphy (1985) which assume an $\alpha / \mathrm{p}$ abundance ratio of 0.1 . As shown by the results of Table 5, the total 4-7 MeV radiation yield is only slightly dependent on the proton spectral index.

Comparing the values of $N_{p>30} \mathrm{MeV}$ indicated in Table 5 and those indicated in Tables 1 to 4 lead to the following conclusions:

- A hard proton spectrum $(\delta=2)$ extending from a few MeV to $E_{\max }$ is not consistent with all the $\gamma$-ray observations. Indeed, the number of protons deduced from Table 5 is much too large compared to the one deduced from pion radiation.

- An intermediate proton spectrum $(\delta=3)$ from a few $\mathrm{MeV}$ to $E_{\max }$ may be consistent with all the $\gamma$-ray observations for spectra 2 to 4 (i.e. from the second $\gamma$-ray peak) only in the case of a high energy cut-off of $750 \mathrm{MeV}$.

- A steep proton spectrum $(\delta=4)$ extending to $E_{\max }$ without hardening is consistent with all the $\gamma$-ray observations of spectra 1 to 2 (i.e. spectra with no predominant pion contribution) if $E_{\max }$ greater than $2 \mathrm{GeV}$ but produces only roughly half of the observed pion radiation of spectra 3 and 4 .

\section{Contribution of the accelerated helium}

As recalled in the introduction, some recent results from $\gamma$-ray observations have led to the conclusion that the accelerated $\alpha / \mathrm{p}$ ratio can be as high as 0.5 in some flares. Given our limited number of observational constraints, we shall only discuss in the following how the consideration of accelerated helium may modify our conclusions. An analysis of photon spectra similar to the one described in Sects. 4 and 5.2 was done now considering total pion decay radiation spectra produced both by protons and $\alpha$ particles with $\alpha / \mathrm{p}=0.5$. It is then found that due to the higher cross sections at low energies of the $\alpha$-p pion production reactions, the inclusion of $\alpha$ particles with $\alpha / \mathrm{p}=0.5$ may lead to a reduction by a factor as large as 2 (in particular in the cases of steep spectra) of the total number of energetic ions (protons and alphas) necessary to produce the observed radiation. The inclusion of $\alpha$ particles will of course also modify the yield of 4 to $7 \mathrm{MeV}$ prompt $\gamma$-lines especially for steep spectra. The low energy thresholds of cross sections for e.g. 4.4 and 6.2 MeV lines are indeed lower by a few $\mathrm{MeV} /$ nuc for line production by $\alpha$ 's compared to line production by protons. However the effects of including $\alpha$ particles will be less important than for pion production since the difference in magnitudes of cross sections for $\alpha$ and proton productions is smaller. Even if accelerated helium is important in the present flare, and may help reduce the discrepancies between the $\gamma$-ray line and pionproducing ions, there will be still some difficulties (the discrepancy is more than a factor 2) suggesting that the energetic ion spectrum in the flare is not a simple power law extending without breaks or changes in spectral shapes from the $\gamma$-ray line emitting domain (a few $\mathrm{MeV} /$ nuc to a few tens of $\mathrm{MeV} /$ nuc) to the pion producing domain (above a few hundreds of $\mathrm{MeV}$ ). It must be noted that a similar remark was made by Kocharov et al. (1996) based on the comparison of solar neutrons and 2.2 MeV line fluence for the same flare.

\section{Discussion}

In the following we discuss the extent to which GRL intensities and deka-MeV continuum may be combined to yield a consistent picture of flare ion acceleration in this event, at least in terms of the parameters $N_{p>30 \mathrm{MeV}}, \delta$ and $E_{\max }$. With the present data, however, the deka-MeV continuum allows the delineation only of acceptable regions of parameter space. Only at photon energies well above the $100 \mathrm{MeV}$ maximum of PHEBUS/GRANAT would the shape of the pion continuum directly reflect the injected ion distribution (Dermer 1986a); this leaves the intensity of the continuum in the region of the $\pi^{0}$ maximum at $67 \mathrm{MeV}$ as the only observed quantity constraining these three parameters. Ambiguity in the origin of any underlying, monotonic continuum merely underlines this situation (as was assumed to be the case by Alexander et al. 1994).

The weak energy-dependence of excitation line crosssections results in values of $N_{p>30} \mathrm{MeV}$ which do not depend strongly on the assumed value of $\delta$, letting us reach the conclusions of the previous section. We may summarise these as follows: the GRL and deka-MeV continuum may be simultaneously interpreted in terms of a single power-law ion energy distribution only for $\delta \sim 3$, and $E_{\max }$ around $0.7 \mathrm{GeV}$. Such a low value of the high energy cut-off of the ion spectrum is however inconsistent with what was deduced from neutron observations (Debrunner et al. 1997). If $\delta$ characterising the 10 $100 \mathrm{MeV}$ energy distribution is less than 3, either the distribution must soften towards $E>300 \mathrm{MeV}$, or we must entertain even smaller values of $E_{\max }$ (already suggested in Alexander et al. 1994). On the other hand, if the ion energy distribution in the $10-100 \mathrm{MeV}$ range is softer than that described by $\delta \sim 3$, we must conclude, that later in the event the distribution hardens at higher energies, in order to produce

enough deka-MeV continuum. In particular, if we wish to insist on $E_{\max } \sim 2 \mathrm{GeV}$ in periods 3 and 4 , as suggested by 
neutron monitor data (Debrunner et al. 1997), we would do this by assuming a distribution characterised by $\delta \sim 4$ in the 10$100 \mathrm{MeV}$ range, hardening to $\delta \sim 3$ in the $0.3-2 \mathrm{GeV}$ energy range. Earlier in the flare, all the deka-MeV continuum may be interpreted as primary electron bremsstrahlung, and constraints from those times are only upper limits, statements of how much pion decay emission we can "hide" in the observed, featureless continuum. The deduction of ion numbers here has assumed an accelerated $\alpha / \mathrm{p}$ abundance ratio of 0.1 .

Taken together, the data analysed here seem to point to an ion energy distribution which does not have simple power-law form. This was already suggested for this flare by Kocharov et al. (1996) based on the comparison of solar neutrons and 2.2 MeV line fluence. They however found very flat spectra for the low energy part of the ion spectrum as well as a steepening of the spectrum towards high energy which is contradictory with what is found here. This may due to the use in their work of gamma-ray line fluences not corrected from instrumental non linear effects. Different findings have also been reported in the literature for other events. On one hand, Dunphy et al. (1999) found that a single power law for the ion distribution in the 11 June, 1991 flare could reproduce both the $\gamma$-ray line and pion decay radiations in the delayed phase. On the other hand, Murphy et al. (1987) found that the ion distribution must fall off with energy faster than a single power-law in the 27 April, 1980 flare. Kocharov et al. (1998) combining GAMMA-1 observations of continuum up to $1 \mathrm{GeV}$ with Comptel observations of flare neutrons and GRLs for the flare of 15 June, 1991 found an under-production of neutrons, compared to that expected by extrapolating the GRL-deduced ion distribution into the neutron production energy range, and an over-production of very high-energy $\gamma$-ray continuum, compared to the extrapolation upwards in energy of the neutron-deduced ion distribution. They interpreted the totality of these observations in terms of an ion distribution that softened at $\sim 300 \mathrm{MeV}$, and hardened again at $\mathrm{GeV}$ energies. With a more complete treatment of the Comptel neutron response, Toner et al. (2001) found a smaller value for the total number of produced neutrons, thus implying an even more pronounced steepening around $300 \mathrm{MeV}$ than that deduced by Kocharov et al.

Flare-integrated ion numbers found in this paper from the nuclear line fluence are in the region of $N_{p>30 \mathrm{MeV}} \sim 4 \times 10^{33}$ (spectra 1 to 4 with $\delta=3$ ) in agreement with the lower limit deduced from the 2.2 MeV line in Debrunner et al. (1997), but a factor of two lower than the number deduced from the neutrons in the same paper. We note that an abundance enhancement of accelerated $\alpha$ 's, from $\alpha / p=0.1$ to 0.5 , suggested in other events from studies of $\mathrm{Li}$ and Be formation lines (Share \& Murphy 1998), would at least double the neutron yield (Murphy et al. 1997; Hua et al. 2002) potentially resolving any discrepancy.

\section{Conclusions}

The large flare of 24 May 1990 (GOES X9.3) produced strong $\gamma$-ray line emission and $\gamma$-ray continuum above $10 \mathrm{MeV}$ predominantly coming from pion decay radiation from the rise of the second peak of the impulsive phase. It was also one of the largest solar neutron events observed so far. These obervations allow one to analyse whether it is possible to infer a consistent picture of flare ion acceleration for energies going from a few $\mathrm{MeV}$ to $\mathrm{GeV}$. The main conclusions that can be drawn from the quantitative analysis of the high energy continuum are the following ones:

- The lack of spectral information above $100 \mathrm{MeV}$ does not allow one to unambiguously determine the primary proton energy spectrum above $300 \mathrm{MeV}$, in particular the maximum proton energy. Only acceptable regions of the proton distribution parameters are delineated: the number of protons (above $300 \mathrm{MeV}$ ) for an assumed proton maximum energy and spectrum.

- Using the additional information provided by the $\gamma$-ray line observations as well as some constraints from the neutron observations, it is found that the ion energy distribution cannot be a single power law from the $\mathrm{MeV}$ to the $\mathrm{GeV}$ range.

It is clear from this analysis that detectors operating at significantly higher energies than PHEBUS will be needed if we wish to constrain further the extremes of solar ion acceleration. An upper cutoff in accelerated ion energies implies an upper cutoff in emitted photon energies. It appears that this will generally lie in the photon energy range above $300 \mathrm{MeV}$, so that direct measurements of it would effectively remove one of the degrees of freedom $\left(E_{\max }\right)$ present in this study. Finer spectral resolution in the $10-200 \mathrm{MeV}$ energy range might allow a clearer determination of the magnitude of the $\pi^{0}$ feature above the otherwise monotonic continuum; estimates of source magnetic field strength could result, and any determination of its detailed shape would yield further information on ion energy distribution, including possibly $E_{\max }$. This study has also demonstrated - again - the value of energy distribution diagnostics from GRL's (particularly when spectral resolution is adequate to allow good measurements of lines that depend to different degrees on fast protons and $\alpha$ 's - (Share \& Murphy 1995, 1998), and a capability for neutron spectroscopy, which provides an invaluable further window on ion acceleration to several $100 \mathrm{~s}$ of $\mathrm{MeV}$ energy, as well as allowing further comment on accelerated $\alpha / \mathrm{p}$ abundance ratios.

Acknowledgements. This work has been facilitated by travel support from the British Council Alliance programme. ALM acknowledges the computational facilities provided by the Starlink Project which is run by CCLRC on behalf of PPARC.

\section{References}

Akimov, V. V., Afanasev, V. G., Belonsov, A. S., et al. 1992, Sov. Astron. Lett., 18, 69

Alexander, D., Dunphy, P. P., \& MacKinnon A. L. 1994, Sol. Phys., 151,147

Barat, C., Trottet, G., Vilmer, N., et al. 1994, ApJ, 425, L109

Chupp, E. L. 1984, ARA\&A, 22, 359

Chupp, E. L. 1996, in High Energy Solar Physics, ed. R. Ramaty, N. Mandzhavidze, \& X.-M. Hua (New York: AIP), AIP Conf. Proc., 374,3

Debrunner, H., Lockwood, J., \& Ryan, J. M. 1992, ApJ, 387, L51 
Debrunner, H., Lockwood, J., \& Ryan, J. M. 1993, ApJ, 409, 822

Debrunner, H., Lockwood, J. A., Barat, C., et al. 1997, ApJ, 479, 997

Dennis, B. R. 1988, Sol. Phys., 118, 49

Dermer, C. D. 1986a, A\&A, 157, 223

Dermer, C. D. 1986b, ApJ, 307, 47

Dunphy, P. P., Chupp, E. L., Bertsch, D. L., et al. 1999, Sol. Phys., 187,45

Forrest, D. J., Vestrand, W. T., Chuppp, E. L., et al. 1985, Proc. 19th ICRC, 4, 146

Kanbach, G., Bertsch, D. L., Fichtel, C. E., et al. 1993, A\&AS, 97, 349

Kocharov, L. G., Lee, J. W., Zirin, H., et al. 1994, Sol. Phys., 155, 149

Kocharov, L., Kovaltsov, G., Torsti, J., et al. 1996, in High Energy Solar Physics, ed. R. Ramaty, N. Mandzhavidze, \& X.-M. Hua (New York: AIP), AIP Conf. Proc., 374, 246

Kocharov, L., Debrunner, H., Kovaltsov, G., et al. 1998, A\&A, 340, 257

Koval'tsov, G. A., Kocharov, G. E., Kocharov, L. G., \& Usoskin, I. G. 1994, AstL, 20, 658

Hua, X.-M., \& Lingenfelter, R. E. 1987, Sol. Phys., 107, 351

Hua, X. M., Kozlovsky, B., Lingenfelter, R. E., Ramaty, R., \& Stupp, A. 2002, ApJS, 140, 563

Mandzhavidze, N., \& Ramaty, R. 1992, ApJ, 389, 739

Mandzhavidze, N., Ramaty, R., \& Kozlovsky, B. 1997, ApJ, 489, L99

Mori, M. 1997, ApJ, 478, 225
Murphy, R. J. 1985, Ph.D. Thesis, University of Maryland

Murphy, R. J., Dermer, C. D., \& Ramaty, R. 1987, ApJS, 63, 721

Murphy, R. J., Share, G. H., Grove, J. E., et al. 1997, ApJ, 490, 883

Ramaty, R. 1986, in Physics of the Sun, ed. P. A. Sturrock, et al. (Dordrecht: D Reidel Publishing Company), 291

Ramaty, R., Mandzhavidze, N., \& Kozlovsky, B. 1996, in High Energy Solar Physics, ed. R. Ramaty, N. Mandzhavidze, \& X.-M. Hua (New York, AIP), AIP Conf. Proc., 374, 172

Share, G. H., \& Murphy, R. J. 1995, ApJ, 452, 933

Share, G. H., \& Murphy, R. J. 1998, ApJ, 508, 876

Stecker, F. 1970, Ap\&SS, 6, 377

Talon, R., Trottet, G., Vilmer, N., et al. 1993, Sol. Phys., 147, 137

Terekhov, O. V., Sunyaev, R. A., Kuznetsov, A. V., et al. 1993, AstL, 19,65

Toner, M. P., Ryan, J. M., MacKinnon, A. L., et al. 2001, in Recent Insights into the Physics of the Sun and Heliosphere: Highlights from SOHO and Other Space Missions, ed. P. Brekke, B. Fleck, \& J. B. Gurman, IAU Symp., 203, 573

Torsti, J., Kocharov, L. G., Vainio, R., et al. 1996, Sol. Phys., 166, 135

Trottet, G., \& Vilmer, N. 1997, Lecture Notes in Physics, 489, 219

Vilmer, N., \& MacKinnon, A. L. 2003, in Energy Conversion and Particle Acceleration in the Solar Corona, ed. L. Klein (Springer), 127 